NuCLEAR reactions often give us very direct information about the detailed shell structure of nuclei. This is shown particularly well by the many studies of single-particle states by one-nucleon transfer reactions. In these reactions, a nucleon is either removed from a nucleus or given to it, and study of the angular distribution of the outgoing particles tells us the quantum numbers and the occupation probabilities of the singleparticle state involved (Nature, 245, $74 ; 1973 ; 249,695 ; 1974)$.

There are also some reactions in which more than one nucleon is transferred at the same time, and these can sometimes tell us about the nucleons that are not participating directly in the reaction. Such

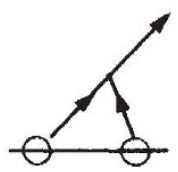

Filled

Shells

No blocking

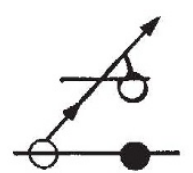

Filled

Shells

Blocking
Neutron
pair removed

\section{- Blocking} neutron

\section{Neutron blocking in alpha-transfer}

\author{
from $P$. E. Hodgson
}

nucleons can get in 'the way, or 'block', the reaction, thus reducing the probability of its taking place.

The first examples of this were found in studies of the $(p, t)$ reaction, which removes two neutrons from the nucleus. In a nucleus with an even number of nucleons both neutrons can be removed from the same singleparticle state, whereas if there is an odd number of neutrons it may be necessary to take the neutrons from different states. This latter process is more difficult, and the cross section for the reaction is reduced by a factor of about two. In this way a neutron that does not participate directly in the reaction nevertheless makes its presence felt and thus gives us information about nuclear structure (Fleming, Blann and Fulbright, $\mathrm{Nucl}$. Phys., A163, 401; 1971).

This blocking effect has now been observed in alpha-transfer reactions as well. Becchetti and Jänecke (Phys. Rev. Lett., 35, 268; 1975) have measured the cross sections of several $\left(d,{ }^{6} \mathrm{Li}\right)$ reactions on even and odd isotopes of tin. The reactions on the even isotopes can just remove the two neutrons from one single particle state and the two protons from another, but the reactions on the odd isotopes have to take the neutrons from different states. This is more

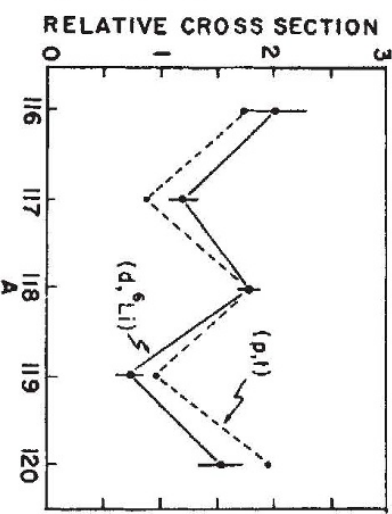

Comparison between the relative cross sections of $(\mathrm{p}, \mathrm{t})$ and $\left(\mathrm{d},{ }^{6} \mathrm{Li}\right)$ reactions as a function of $A$ for some tin isotopes.

difficult, and as shown in the figure the corresponding cross sections are again reduced by a factor of about two.

This blocking in alpha-transfer is very similar to the same effect in $(\mathrm{p}, \mathrm{t})$ reactions, and suggests that the mechanism is essentially the same, with the two transferred protons acting simply as spectators. The regularity of the reduction suggests that it is the $3 S_{1 / 2}$ state that has an odd nucleon in the odd isotopes, and this is expected from the simple shell model.

The spectroscopic factors obtained by analysing the data with the distorted wave theory show a similar reduction for the odd isotopes, confirming that it is not simply due to the differences in binding energies and $\mathrm{Q}$-values, but is a real nuclear structure effect. gives $T=4.3 \times 10^{5} \alpha r^{2} M^{-\frac{2}{3}}$. If the body is assumed to have a constant density, $\rho$, throughout (that is, $M=1.33 \pi r^{3} \rho$ ) and an $\alpha$ of 0.4 then $T=6.59 \times 10^{4} \rho^{-\frac{2}{3}}$, which, when the assumption is made that all Solar System bodies have the same density and no central mass concentration, confirms Alfven's findings (Icarus, 3, 52; 1964) that all planets and asteroids have approximately the same period of rotation. Using a density of $3 \mathrm{~g} \mathrm{~cm}^{-3}$ this period comes to $8.8 \mathrm{~h}$.

It can be seen from Fig. 1 that Mercury and Venus have considerably lower angular momenta than predicted by the linear relationship. This is due to tidal interactions between these planets and the Sun. The high rate of spin deceleration suffered by Venus indicates that the solid dissipation inside the planet is considerable. This is only possible if large regions in the interior of Venus are near their melting point thus enabling creep, elastoviscosity and direct viscous interactions to play their part in dissipating energy. Combining the angular momentum of the spinning Earth and the orbital angular momentum of the Moon gives a point on the line in Fig. 1 indicating that the angular momentum of the Earth-Moon system has not changed much during the history of the Solar System. All that has happened is that the Moon has moved further away, and the Earth now spins more slowly.

In a recent paper in Icarus $(\mathbf{2 5}, \mathbf{5 4 5}$; 1975) Burns doubles the number of asteroid points used in Fig. 1. Accurate sizes based on the radiometry and polarimetry work of Chapman, Morrison and Zellner (Icarus, 25, 104; 1975) are used together with an attempt to include for the first time the effect of an asteroid's non-sphericity. The brightness of asteroids vary with periods scattered around $9 \mathrm{~h}$. These brightness variations, which in some instances range up to two magnitudes but are more typically 0.2 magnitude, are primarily caused by the asteroid having an irregular shape and have been used by Burns to deduce their shape. As the asteroids usually rotate about their principal axes of maximum moment of inertia they contain more angular momentum per unit mass than a spherical body. The effect of interasteroid collisions should make the smallest asteroids spin faster and be more irregular. It can be seen in Fig. 1 that 1566 Icarus and 1620 Geographos both lie above the straight line, indicating that they are spinning faster than expected.

Why is the spin period around $9 \mathrm{~h}$ ? It seems that the accretion processes which formed the planets and asteroids become less and less efficient as these bodies get nearer and nearer to their rotational stability limit. This limit occurs when the rotational surface velocity of the body is equal to its escape velocity (that is when $\mathrm{T} \approx 3.3 p^{-\frac{1}{2}} \mathrm{~h}$, a rotational period of $\sim 2 \mathrm{~h}$ for a body of density $3 \mathrm{~g} \mathrm{~cm}^{-3}$ ). A conclusion might be that bodies cannot accrete if they spin faster than about one third the rotational stability limit. 